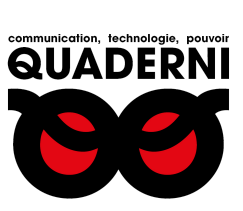

\title{
Quaderni
}

Communication, technologies, pouvoir

96 | Printemps 2018

Smart city : "fiction" et innovation stratégique

\section{Smart City :"fiction" et innovation stratégique}

Avant-propos

Cynthia Ghorra-Gobin

\section{(2) OpenEdition}

Journals

Édition électronique

URL : https://journals.openedition.org/quaderni/1169

DOI : 10.4000/quaderni.1169

ISSN : 2105-2956

Éditeur

Les éditions de la Maison des sciences de l'Homme

Édition imprimée

Date de publication : 15 mai 2018

Pagination : 5-15

Référence électronique

Cynthia Ghorra-Gobin, «Smart City :"fiction" et innovation stratégique », Quaderni [En ligne], 96 |

Printemps 2018, mis en ligne le 15 mai 2018, consulté le 28 septembre 2021. URL : http://

journals.openedition.org/quaderni/1169; DOI : https://doi.org/10.4000/quaderni.1169 


\section{$D$ ossier}

\section{Smart City : "fiction" et innovation stratégique}

Ce dossier de Quaderni propose de croiser différentes approches de sciences humaines et sociales sur la ville intelligente. Il a pour objectif de questionner le slogan 'smart city' utilisé non seulement par des ingénieurs des techniques numériques mais aussi par des responsables politiques, des professionnels

\section{Cynthia Ghorra-Gobin}

Géographe, CNRS-CREDA, Université Sorbonne Nouvelle Paris 3 de l'aménagement urbain et des chercheurs. Il tente de répondre à la question : "la smart city » relève-t-elle d'une " fiction» (e-democracy au-delà de e-government) au service d'une innovation technique - qui tout compte fait se limite à l'optimisation de services urbains (grâce aux capteurs, aux objets connectés et à Internet) - proposée par des entreprises privées ou fautil plutôt l'interpréter comme une innovation majeure autorisant une gestion stratégique de la ville en cours de recomposition spatiale, sociale et économique sous l'effet de la mondialisation et de la révolution numérique?

Les villes se recomposent sous l'effet de la révolution numérique et de la métamorphose du capitalisme globalisé et financiarisé. Elles sont devenues des sites stratégiques pour l'action publique, pour les entreprises et parfois pour des mobilisations sociales et politiques'. À l'heure des flux (capitaux, touristes, classes créatives et marchandises), les responsables politiques ont pour ambition première d'en assurer l'attractivité. Leurs programmes se traduisent par la construction de clusters (pôles de compétitivité), de mégaprojets, d'infrastructures en vue d'une mobilité douce (tramway, vélo, marche à pied) susceptibles de s'accompagner d'opérations de patrimonialisation ainsi que d'équipements culturels prestigieux (musées, hubs culturels, festivals, programmes d'animation urbaine). Ces 
opérations d'envergure - qui exigent souvent de lourds investissements - sont critiquées par ceux qui dénoncent la politique néolibérale des villes, et défendent les laissés-pour-compte. Dans le but de faire face à ces critiques, des expressions ont été inventées pour orienter sur un autre mode l'action publique. On parle ainsi de « ville inclusive» (inclure les exclus de la mondialisation), « ville frugale » (diminuer l'empreinte écologique, la production de gaz à effet de serre), « ville juste » (lutter contre les inégalités sociales, spatiales et environnementales), « ville durable » (prendre en compte les objectifs du développement durable), « ville verte » ou « ville résiliente » (affronter toute forme de vulnérabilité dont celle liée au changement climatique) et plus récemment « ville intelligente » (VI) ou « smart city $»(\mathrm{SC})$ et parfois « resilient smart city ».

L'expression « ville intelligente » est volontairement utilisée en anglais dans ce dossier. Il sera ainsi question de Smart City (SC), ce qui permet de situer sur le mode explicite le contexte américain de son émergence, c'est-à-dire l'univers des entreprises high-tech de la Silicon Valley - souvent désignées par le sigle GAFA (Google, Apple, Facebook, Amazon) - qui ont la prétention d'inventer le devenir urbain et d'assurer l'avènement d'une l'humanité connectée ${ }^{2}$. La $\mathrm{SC}$ peut renvoyer à l'optimisation technique du fonctionnement de la ville et de l'offre de services urbains grâce à la maîtrise de données numériques (dans des domaines aussi variés que la transition énergétique, la mobilité, la qualité de l'air). Mais elle véhicule également le message de l'émergence de l'intelligence collective (plutôt que de l'intelligence artificielle), du civisme numérique (digital civics), de l'instauration d'une relation directe entre l'élu et ses administrés (e-democracy), bref du « vivre-ensemble ».

L'appellation SC ne fait pas uniquement référence à une représentation de la ville pensée comme un système de « flux numérisés » (Goldsmith \& Crawford, 2014) ${ }^{3}$, elle donne à voir des individus capables de produire des « externalités positives » parce que conscients et désireux d'améliorer la qualité de leur environnement. Les usagers opteraient en quelque sorte pour une consommation raisonnée des ressources non renouvelables. La SC est alors perçue comme l'instrument susceptible de favoriser l'émergence d'une qualité de vie qualifiée de responsable. En d'autres termes la technologie n'est plus uniquement envisagée comme un indicateur de l'intelligence d'un système d'information (grâce à des algorithmes puissants et subtils) mais comme un outil au service d'un projet collectif qui se dessinerait à l'échelle mondiale. En s'inspirant des travaux de Lucien Sfez dont la contribution majeure réside dans la portée symbolique de la technique et de la politique, ce dossier interroge la SC comme nouvelle « fiction urbaine ». La citation d'une phrase de l'article publié dans la Revue européenne de sciences sociales (2002) résume bien le positionnement du politiste $\mathrm{Sfez}$ : « La fiction (celle du progrès) exerce une séduction dont nous sentons nous-mêmes les effets dans notre vie quotidienne. C'est la fiction, croyons-nous, qui est le moteur de la technique, et par là de la politique. ${ }^{4}$ »

Aussi ce dossier interprète la SC comme une figure oscillant entre fiction et innovation numérique qualifiée de stratégique pour la condition urbaine. Il propose le questionnement suivant : 
comment expliquer la diffusion rapide de la thématique de la « smart city » dans les médias, les institutions et la recherche en sciences sociales? S'agit-il d'une technique susceptible de produire des données intelligentes de manière à concilier efficacité économique (fluidité de la mobilité) et mieux-être social (mobilité douce) ? Ne se présente-t-elle pas alors comme un programme participant du marketing territorial de la ville et son branding en raison de la publicité présentée dans les médias ? Ne faut-il pas plutôt considérer la SC comme la révélation de la condition urbaine contemporaine et ainsi comprendre la 'mutation métropolitaine' qui est en train de s'opérer? Peuton se rallier à ceux qui imaginent la SC comme l'avènement d'un « régime urbain collaboratif » parce qu'il associe élus, habitants, usagers et opérateurs de réseaux ? Dans ce cas, ne peut-on déceler l'élaboration d'une « fiction » suggérant la refondation d'une société « éclatée »?

Après avoir rappelé de récentes manifestations médiatiques ainsi que l'introduction de programmes institutionnels de recherche et d'enseignement concernant la SC dans notre contexte national, ce dossier de Quaderni tente de répondre au questionnement proposé en croisant différentes approches de sciences humaines. La diffusion de la SC n'interroge pas uniquement les ingénieurs des techniques numériques. Des responsables politiques, des professionnels de l'aménagement urbain et de chercheurs en sciences sociales tentent d'y répondre. Le dossier privilégie l'hypothèse de la SC comme l'illustration d'une « fiction » urbaine et sociale (e-democracy au-delà de e-government) au service d'une innovation technique. Certains, à la suite d'Antoine Picon', parlent de l'« utopie collaborative » et d'autres, à la suite d'Anthony Townsend ${ }^{6}$, parlent de la « nouvelle utopie ». Mais un système technique proposé par des entreprises dont l'objectif est d'optimiser l'offre de services urbains (grâce aux capteurs, objets connectés et Internet) peut-il aller jusqu'à « réenchanter la démocratie ? ${ }^{7}$ »

\section{Le contexte médiatique}

La ville intelligente est présentée par les médias comme une « innovation » technique autorisant le fonctionnement optimal de la ville parce que reposant sur une gestion en temps réel de données fournies par les gestionnaires des équipements et par les usagers. Les données des usagers sont aisément captées parce qu'ils sont quasi connectés et géolocalisés par le biais des ordinateurs, des téléphones et des objets intelligents. Il est question d'Open Data ${ }^{8}$ (accès aux données) et de Big Data (statistiques de masses). L'accent est mis sur la technique comme moyen pour optimiser la gestion de la ville et de ses flux. Certains médias et professionnels de l'aménagement urbain vont jusqu'à évoquer des images d'échanges et de dialogues fructueux entre décideurs et usagers au sujet des futurs projets d'aménagement, grâce à des applications mises au point par des start-ups.

Les médias français ont largement évoqué la $51^{\mathrm{e}}$ édition du Consumer Electronic Show (CES) de Las Vegas (Nevada) qui pour la première fois, a consacré un espace d'exposition et un programme de conférences à la ville intelligente entre le 9 et le 12 janvier 2018. Ils ont reconnu que le CES avait du retard par rapport au Smart City Expo World Congress, organisé chaque année à Barcelone depuis 2011. Ils ont toutefois souligné 
combien la SC fut la « guest star » à côté de la voiture « connectée » (autonome). Désormais l'argument de la connectivité a remplacé celui de l'environnement comme l'indique le slogan des industriels "Smart is the New Green ». Certains journalistes ont regretté l'absence des décideurs publics contrairement au Smart City Expo World Congress de Barcelone où les élus et la société civile sont représentés depuis deux ans déjà.

En soulignant l'importance de la SC, les médias diffusent les chiffres de Consumer Technology Association, organisatrice du Salon de l'électronique grand public, pour montrer l'enjeu qu'elle représente. Le marché mondial des villes connectées devrait ainsi passer de 12 milliards d'euros en 2015 à 29 milliards d'euros en 2020. La perspective d'un marché mondial de la SC est inscrite dans le rapport au Premier ministre sur l'avenir des SC, présenté par le député Luc Belot en Avril 20179. La France offre des services publics de qualité et elle « dispose également d'une expertise reconnue mondialement dans les marchés des services publics locaux (eau, énergie, déchets, notamment) ». D'où le défi présenté aux entreprises françaises pour conserver leur position de leader sur les métiers de la ville dans ce marché mondialisé.

Le quotidien Le Monde (en partenariat avec des entreprises privées) a décerné au cours des deux dernières années, en 2016 et 2017 le Prix Le Monde-Smart Cities à des villes et des start-up ayant fait preuve d'innovation dans les domaines de l'énergie, de l'habitat, de la mobilité et de la participation civique. En 2017 à l'occasion de la remise des prix, Le Monde a organisé une journée d'échanges ouverte au public à Lyon (7 avril) dans les locaux de l'hôtel de ville. Le Prix de l'innovation urbaine a été décerné à Lyon Confluence pour la maîtrise énergétique. Dans ce nouveau quartier de la ville, trois immeubles de l'ensemble Hikari ont été équipés de capteurs qui mesurent la température, détectent le $\mathrm{CO}^{2}$, enregistrent des paramètres qui permettent de réguler en temps réel la production de chaud et de froid et calculent en continu les consommations. Ce qui permet d'optimiser, selon son mode de vie, le rapport entre économie et confort.

Le Monde a également décerné sept Prix mondiaux de l'innovation le 2 juin 2017 à Singapour et a organisé une journée d'échanges ouverte au public avec ses partenaires, la Caisse des Dépôts et Consignations, l'Atelier BNP, Engie, St Gobain, Keolis, Veolia et Enedis. On y note le Prix Participation citoyenne décerné au projet pédestre de Curridabat, une ville du Costa Rica qui a mis au point une application permettant de recenser les obstacles rencontrés dans la rue par des personnes handicapées. Le Prix Habitat a été décerné à Récipro-Cité de Lyon, une entreprise d'ingénierie du lien social qui a mis au point un dispositif qui permet le développement d'activités collaboratives entre les habitants d'un immeuble ou d'un quartier, à travers l'association Chers voisins et une Maison de projets.

L'attribution de ces prix qui mettent bien en évidence la dimension sociale de la SC a fait l'objet d'articles faisant référence aux travaux de Carlo Ratti, le directeur d'un prestigieux laboratoire urbain au sein du MIT (Senseable City Lab). Ce dernier affirme en effet qu'un bouleversement s'opère dans le champ urbain avec l'avènement 
des outils numériques : «depuis qu'Internet se déploie dans l'espace physique l'aménagement urbain ne peut plus se limiter aux seules professions d'architectes et d'urbanistes ». Ratti en déduit que la clé de la réussite urbaine réside désormais dans la co-construction de toutes les parties prenantes de la ville ${ }^{10}$. Ce point de vue converge avec celui développé par des géographes qui dans un dossier de la revue Urban Geography soulignent combien la SC correspond moins au triomphe des techniques numériques qu'à un processus de transformation des villes touchant leur gouvernance et les politiques de l'aménagement urbain ${ }^{11}$. Cette hypothèse est également partagée par Jean-Marc Offner qui (dans l'article du dossier) inscrit la SC dans la mouvance de l'urbanisme des réseaux.

D'autres chercheurs vont plus loin. Ils pensent que cette grande transformation urbaine sous l'effet de la révolution numérique se doublerait d'une interrogation visant à enrayer la crise de défiance politique et civique. La SC est alors perçue comme une voie en mesure de renouveler le sens civique et d'incarner la e-democracy, comme l'évoque l'expérimentation tenue au début de l'année 2017 à Hambourg. Pour accueillir rapidement 50000 migrants et s'assurer l'acceptation de la population, le bourgmestre a piloté une « consultation-participation » préparé par le laboratoire de l'université Hafen-City. Il revenait aux habitants d'identifier la localisation future des centres destinés aux nouveaux résidents. Les médias ont alors présenté la SC comme une transformation de la ville qui se veut créative et fascinante parce qu'elle permet également l'invention de nouvelles façons du « vivre ensemble ».
Les revues d'urbanisme et d'aménagement urbain ont souvent évoqué les projets de « Songdo International Business District » au sud de Séoul en Corée du Sud et de "Masdar » à proximité de l'aéroport international d'Abou Dhabi comme les modèles de l'hyperconnectivité et de l'écoresponsabilité. Un récent numéro de la revue Urbanisme ${ }^{12}$ étudie le projet de SC à Toronto. Il s'agit d'un projet créé par les autorités publiques et Sidewalk Labs, (filiale d'Alphabet, holding de Google) qui contrairement à Songdo et Masdar, inclut les habitants dans la réalisation du quartier connecté. Dans notre contexte national, il est souvent question de l'application Vooter (2015) qui permet aux élus de consulter leurs administrés sur la réalisation d'une infrastructure ou d'un grand projet d'urbanisme. Celle-ci est présentée comme un moyen de simplifier la vie des élus et des habitants parce qu'elle évite d'organiser des réunions publiques. Vooter a été sollicité par plusieurs villes dont Issy-les-Moulineaux et Boulogne-Billancourt. Avec la SC, les ingénieurs parlent $d$ ' « approche collaborative » sur le mode numérique mais ils oublient de préciser combien l'application devient un moyen de supprimer avec subtilité le principe de la «proximité spatiale » dans le dialogue social.

\section{Des programmes de recherche et d'ensei- gnement élaborés par des institutions}

La Chaire de Sciences Po « Mutations de l'action publique et du droit public » (en partenariat avec la maison d'édition Berger-Levrault) a lancé en 2015 le programme « La Cité des smart cities. ${ }^{13}$ » Elle part du principe que l'avènement de l'économie de la connaissance (succédant à l'économie industrielle) se joue désormais dans la ville, un 
phénomène qui exigerait un nouveau type de gouvernance. Il s'agit alors d'assurer le rééquilibrage des rapports juridiques, entre citoyens, start up, opérateurs de réseaux et autorités publiques sur le plan juridique. Aussi le programme est centré sur les modes de gouvernance. En 2017, l'École Urbaine de Sciences Po et le Centre d'études européennes ont inauguré une nouvelle Chaire de recherche et d'enseignement intitulée «Villes et numérique » avec le soutien de quatre mécènes (Cisco, La Poste, RTE, CDC). La Chaire a pour objectif d'étudier l'interaction entre le numérique et les territoires et de centrer l'analyse sur les politiques de données urbaines.

L'École des Ponts et le cabinet KMPG se sont également engagés dans la création d'une chaire "Smart Cities et création de valeur » parallèlement à un des masters intitulé «Ingénierie et management des SC » piloté avec l'École des Ingénieurs de la ville de Paris. Le PUCA (ministère de la Transition écologique) en collaboration avec le centre de recherche Latts-CNRS a lancé un second cycle de réflexion (2017-2018) sur les dimensions politiques de la ville intelligente. Il s'agit de questionner les présupposés de la ville intelligente, ses réalisations techniques ainsi que les transformations spécifiques observées. Le premier cycle qui s'est déroulé entre 2014 et 2016 se voulait exploratoire et avait insisté sur l'articulation entre «ville intelligente » et « développement durable ». La ville intelligente serait une étape indispensable pour assurer le développement de la « ville durable ». Le second cycle met l'accent sur la régulation de la SC, compte tenu de la diversité et de la multiplicité des acteurs, comme l'indique l'article de François Ménard publié dans Urbanisme ${ }^{14}$.
Différentes institutions s'approprient la thématique de la Smart City comme une innovation technique ancrée dans la révolution numérique et susceptible de résoudre les problèmes urbains liés à la mobilité, à la transition énergétique, à la qualité de l'air tout en associant les parties prenantes, soit les usagers. Ce constat converge avec les propositions du Parlement européen qui définit la $\mathrm{SC}$ comme « une ville qui cherche à résoudre les problèmes publics grâce à des solutions basées sur les TIC, sur la base d'une initiative municipale et mobilisant de multiples parties prenantes ». La SC représenterait ainsi une nouvelle étape dans la gouvernance de la ville et de fait transformerait la condition urbaine. Il s'agit de dépasser la simple numérisation des fonctions administratives (e-administration) pour renouer le « dialogue social » parallèlement à l'offre de nouveaux services urbains.

$* * * * *$

Ce dossier de Quaderni sur la SC inclut les articles de cinq chercheurs en sciences sociales dont certains comme Jean-Marc Offner peuvent également être qualifiés d'acteurs professionnels de la ville. Ces articles évoquent : le nouveau regard porté sur la ville au travers de la SC ainsi que sa dimension esthétique ; les controverses soulevées par la SC; l'intérêt que portent les industriels aux échanges avec les élus locaux pour assurer la diffusion de la SC ; la quête d'un maire pour domestiquer la SC; et l'importance de la traduction pour comprendre l'adjectif «smart» de SC.

L'analyse est centrée sur les représentations véhiculées par une $\mathrm{SC}$ non limitée à une inno- 
vation « technique ». Comme indiqué précédemment, les représentations oscillent entre (1) la thèse de la SC comme un discours sur les techniques numériques construit sur le mode d'une «fiction » au nom du « vivre ensemble » et (2) celle de l'émergence d'outils au profit d' une « gestion» qualifiée de « stratégique ».

Pour Jean-Mare Offner qui dirige l'Agence d'urbanisme de Bordeaux et préside le conseil stratégique de l'École urbaine de Sciences Po (Paris), la SC permet de concevoir et de voir la ville sur un autre mode et ainsi de l'appréhender dans ses mutations métropolitaines. Il précise d'emblée que la mise en réseau des territoires (définition de la métropolisation) ne date ni d'Internet ni de la voiture mais de l'urbanisme des réseaux tel qu'il se développe à partir de la fin $\mathrm{du}$ XVIII ${ }^{e}$ siècle. La SC contribue à une meilleure connaissance de la « ville en marche » dans un contexte marqué par l'expertise lacunaire des usages face aux statistiques de stocks (nombre habitants, nombre logements, nombre emplois, $\mathrm{m}^{2}$ de planchers de bureaux...). Dans ce nouveau régime de la ville devenant métropole où la gouvernance consiste à articuler stocks et flux (soit le sédentaire et le mouvement), le smartphone est qualifié d'outil du quotidien des vies mobiles.

Pour le professionnel, la SC présente par ailleurs l'avantage de faire découvrir les plaisirs de la ville, de la proximité et de la densité à des usagers éloignés de la culture de l'urbanité. Elle contribue notamment à donner une visibilité des métabolismes, comme le font les pylônes bordelais du Pont Chaban sur la Garonne. Ceux-ci indiquent par leurs superbes lumières nocturnes quand la marée monte (couleur bleue) et quand elle des- cend (lumière verte). Il s'agit là d'une mise en scène des flux qui traversent la ville. Aussi la SC contribuerait à l'esthétique de la ville.

Le géographe spécialiste des nouvelles technologies, Emmanuel Eveno, appréhende la « ville intelligente » (VI) comme un objet situé au centre de nombreuses controverses. Il commence par expliquer la rapidité de la diffusion de la VI parce que les élus auraient compris qu'en s'appropriant ce titre ou en organisant des évènements sur le thème de la VI, ils assureraient l'attractivité de leurs villes à l'échelle nationale ou encore mondiale. L'auteur insiste sur le rôle des classements internationaux qui permettent aux villes de se mettre en scène à l'échelle mondiale. Il critique les méthodes et critères utilisés par ces professionnels du classement qu'ils soient universitaires ou responsables d'entreprises. Aussi pour Eveno, le label VI connaît un vif succès au même titre que les technopoles dans les années 1990.

Pour le géographe, les controverses autour de la VI proviennent du fait que contrairement à « société de l'information » ou « ville durable», le label n'a pas fait l'objet de rapports officiels équivalents à celui du rapport Nora/Minc en 1976 et du rapport du Club de Rome en 1972. Ces deux rapports présentaient l'intérêt d'assurer l'articulation entre la sphère des chercheurs et celle des décideurs politiques. Le message politique du Club de Rome, Limits to Growth avait été repris lors de la conférence des Nations Unies sur l'environnement humain - organisé la même année à Stockholm - qui avait introduit la notion d' « écodéveloppement ». Eveno en déduit qu'il est pertinent pour la recherche en sciences sociales de s'approprier cette thématique de la VI 
pour limiter malentendus et controverses.

L'article du chercheur Stève Bernardin part de l'hypothèse selon laquelle la SC est associée à une promesse de progrès parce qu'elle est présentée comme un mode opératoire visant à répondre à tous les maux. En s'inscrivant dans une démarche de sociologie des problèmes publics, il propose de centrer son analyse sur l'argumentaire des industriels en faveur de la SC. Il s'intéresse ainsi aux conditions politiques et sociales de production de la SC à partir d'entretiens et d'observations menés à l'occasion d'un travail de terrain dans la Silicon Valley. Il a participé à une conférence de l' « International Society of Service Innovation Professionals » (une association fondée en 2012 par des responsables de trois entreprises du secteur Cisco, IBM et Hewlett-Packard) et discuté avec le directeur des programmes universitaires d'IBM.

Au cours de la conférence, Bernardin a écouté les propos d'un brillant ingénieur ayant fait ses études en Inde qui a affirmé que l'enjeu de la SC n'est pas la technologie mais le « marché de l'emploi » qui risque de changer considérablement ${ }^{15}$. Cette première intervention a été suivie d'une seconde par un ancien responsable politique au niveau de la Californie qui a affirmé que les municipalités et les populations locales étaient prêtes à participer à la $\mathrm{SC}$. Bernardin souligne combien la rencontre - organisée par l'association - était peu centrée sur les technologies numériques en tant que telles et qu'elle marquait surtout l'intérêt des industriels pour dialoguer avec les politiques. L'objectif des industriels consiste à susciter une « science du service » qui viserait au-delà de questions purement académiques à trouver les nouvelles opportunités de création de valeur en vue de créer de « nouveaux modèles économiques ». D'où leur sensibilité au problème de l'adéquation entre l'offre commerciale des industriels et la demande municipale dans la perspective de la « résolution des problèmes urbains » (problem-solving).

Bernardin ne parle pas de « fiction » mais il estime que les industriels partagent avec les collectivités ce projet de citoyenneté renouvelé, qui s'inscrit en fait dans le mythe d'une culture politique ouverte au débat et à l'échange des idées. L'élite industrielle ne peut se passer des interlocuteurs politiques.

Si la relation au politique s'avère indispensable pour saisir les enjeux de la SC, l'entretien mené par le chercheur Kevin Brookes auprès du maire de Grenoble, Éric Piolle, évoque l'exemple d'un élu qui a pris distance avec l'effervescence suscité par la SC. Le choix du maire de Grenoble n'est pas anodin, il s'agit en fait d'un élu écologiste d'un territoire marqué par les secteurs de la recherche et des entreprises de nouvelles technologies. Le maire s'oppose à l'idée d'inscrire la ville dans une logique de flux, pour insister sur la définition de la Cité comme espace de démocratie c'est-à-dire « espace de conflits ». Ce point de vue diffère de son expérience professionnelle dans l'industrie privée (supply chain) où ses responsabilités l'avaient conduit à optimiser la gestion des flux. Le maire insiste pour dire que la SC est en mesure de conduire la société vers un monde d' ' acteurs-réseaux », à condition que le politique s'en mêle. Pour réaliser cet équilibre il propose de travailler simultanément la SC et la ville « low tech ». En s'inspirant de Navi Radjou, il parle 
d'« innovation frugale » qui permet de résister aux chocs comme les cyclones ou les inondations. Il s'agit d'associer technologie et démocratie. On devrait peut-être parler de SC résiliente.

Dans son projet « Grenoble ville de demain », le maire s'est donné pour objectif le principe de la porosité - soit le mélange des individus - dans le cadre de réunions intitulées « fabriques » qui invitent une pluralité d'acteurs à échanger sur un thème travaillé en amont par un professeur et ses étudiants. Dans le cadre de ces réunions, les outils numériques sont mobilisés mais ils ne sont qu'un moyen pour assurer la confrontation et l'échange des idées et des points de vue. Le maire insiste ainsi sur la méthode du bottom up, soit l'émergence de connaissances et d'idées issues du terrain. Dans la conclusion de l'article, Kevin Brookes souligne combien la SC pourrait répondre à des enjeux de justice sociale, si le politique le souhaite.

Le dossier se termine avec l'article du chercheur Marc Chopplet qui revient sur le discours de la ville intelligente et évoque le paradoxe entre (1) le projet des entreprises GAFA en vue de créer un univers d'individus connectés tout en s'inscrivant dans une logique néolibérale et (2) le recours aux outils de la SC pour moderniser le service public en France. Il ne s'attarde pas trop sur ce paradoxe pour centrer l'analyse sur les différences d'interprétation et de définition données par la traduction de l'anglais au français ainsi que sur le sens accordé à l'intelligence par le philosophe John Dewey (1859-1952). Pour les dictionnaires en langue française, l'intelligence est associée à une faculté personnelle d'analyse et de synthèse dissociée de l'action, comme l'esprit le serait du corps. Pour Dewey et pour le pragmatisme américain, l'intelligence renvoie à une capacité à « estimer les possibilités propres à une situation et à agir en fonction de cette estimation $»$. La VI s'inspirerait de cette définition de l'intelligence, puisqu'elle préconise anticipation et estimation de l'« agir » possible.

Avec Chopplet, le lecteur sort du discours de fascination à l'égard de l'outil numérique pour s'inscrire dans une problématique de l'action et du changement, c'est-à-dire dans une perspective politique. L'auteur se demande toutefois si cette ville dominée par les sciences de l'ingénieur ne «flirte » pas avec les imaginaires de la sciencefiction dans la mesure où elle ignore ce qui se passe dans les espaces publics de la ville (les pauvres et les sans-abris) pour ne se concentrer que sur les « hubs ", associés à la mobilité. L'article s'achève sur l'impératif de penser la démocratie qui à l'instar de John Dewey n'est pas un état à préserver mais une construction « sans cesse renouvelée ». 
$\mathrm{N} \cdot \mathrm{O} \cdot \mathrm{T} \cdot \mathrm{E} \cdot \mathrm{S}$

1. Consulter Cynthia Ghorra-Gobin (dir.), Dictionnaire critique de la mondialisation, Armand Colin, 2012 ainsi que La métropolisation en question, PUF, coll. « La ville en débat », 2015.

2. Voir l'introduction d'Antoine Loubière du dossier «Qui gouverne la smart city? », Urbanisme, n 407, hiver 2017.

3. Stephen Goldsmith \& Susan P. Crawford, The Responsive City: Engaging Communities through Data Governance, Jossey-Bass, 2014.

4. Lucien Sfez, "La technique comme fiction», Revue Européenne des Sciences Sociales, 2002, p. 65-74 et Technique et idéologie, un enjeu de pouvoir, Seuil, 2002.

5. Antoine Picon, Smart Cities: Théorie et critique d'un idéal auto-réalisateur, Édition B2, 2013.

6. Anthony Townsend, Smart Cities-Big Data, Civic Hackers, and the Quest for a New Utopia, W.W.Norton \& Company, 2013 et Julien Damon, « La Smart cité en 2040 : une utopie urbaine en deux fictions ", Responsabilité et Environnement, $\mathrm{n}^{\circ} 84$, octobre 2016, p. 5-9

7. Cahier $d u$ Monde (villes intelligentes) $\mathrm{n}^{\circ} 22467$, 7 avril 2017.

8. Voir le site de la mission Etalab pour la modernisation de l'action publique : www.data.gouv.fr

9. Luc Belot, « De la smart city au territoire d'intelligence(s) », Rapport remis au Premier ministre en avril 2017.

10. Cahier $d u$ Monde (villes intelligentes) $n^{\circ} 22511$, 30 mai 2017.

11. Voir Alan Wiig \& Elvin Wyly, «Introduction : Thinking through the Politics of the smart city », Urban Geography, 37 :4, p. 485-493, 2016, dossier "Thinking the Smart City: Power, Politics, and Networked Urbanism".

12. Jean-François Soupizet, « À Toronto, Alphabet relance la smart city », Urbanisme $\mathrm{n}^{\circ}$ 407, p. 37, 2017. Voir également Nicolas Douay, L'urbanisme à l'heure du numérique, Iste Éditions, 2018.

13. Ce programme a fait l'objet d'une publication Smart Cities : L'innovation au cour de l'action publique, Berger - Levrault, 2016.

14. François Ménard, «Penser la ville intelligente », Urbanisme $\mathrm{n}^{\circ}$ 407, p. 32-36, 2017.

15. Cette question de l'emploi est une préoccupation de l'économiste Daniel Cohen. Voir l'entretien qu'il donne à Pour la Science, nº 98, 2018, p. 8-11 dans le cadre d'un dossier sur les « Big Data : vers une révolution de l'intelligence ». 


\title{
$R \cdot E ́ \cdot S \cdot U \cdot M \cdot E ́$
}

Ce dossier de Quaderni sur la smart city (ville intelligente) se situe à l'intersection de différentes approches de sciences humaines et sociales. Il a pour objectif de questionner le slogan 'smart city' utilisé non seulement par des ingénieurs des techniques numériques mais aussi par des responsables politiques, des professionnels de l'aménagement urbain et des chercheurs. Il tente de répondre à l'interrogation suivante : la 'smart city' relève-t-elle d'un récit de « fiction » (e-democracy au-delà de e-government) au service d'une innovation technique - qui tout compte fait se limite à l'optimisation de services urbains (grâce aux capteurs, aux objets connectés et à Internet) proposée par des entreprises privées ou faut-il plutôt l'interpréter comme une innovation majeure autorisant la fluidité de la ville en cours de recomposition spatiale, sociale et économique sous l'effet de la mondialisation et de la révolution numérique?

\begin{abstract}
This special issue of Quaderni on the smart city (ville intelligente) lies at the intersection of different social science approaches. It questions the buzzword smart city not only used by engineers of the digital revolution but also by political officials, planners and researchers. It tries to answer the following interrogation: Does the smart city belong to a "fiction" storytelling (e-democracy beyond e-government) used for a technical innovation - which is actually limited to the optimization of urban services - offered by private firms? Or does it stand up as a major innovation enabling the fluidity of the city involved in a process of spatial, social and economic reconfigurations linked to globalization and the digital revolution?
\end{abstract}


